\title{
Impact of prone position on outcomes of COVID-19 patients with spontaneous breathing
}

\author{
Mabrouk Bahloul ${ }^{1}$, Sana Kharrat ${ }^{1}$, Malek Hafdhi ${ }^{1}$, Anis Maalla ${ }^{2}$, Olfa Turki ${ }^{1}$, Kamilia Chtara ${ }^{1}$, Rania Ammar ${ }^{1}$, \\ Basma Suissi ${ }^{2}$, Chokri Ben Hamida ${ }^{1}$, Hedi Chelly ${ }^{1}$, Khaiereddine Ben Mahfoudh ${ }^{2}$, Mounir Bouaziz ${ }^{1}$ \\ Departments of ${ }^{1}$ Intensive Care and ${ }^{2}$ Radiology, Habib Bourguiba University Hospital and Sfax University, Sfax, Tunisia
}

Background: In this study, we explored whether early application of the prone position (PP) can improve severe hypoxemia and respiratory failure in coronavirus disease 2019 (COVID-19) patients with spontaneous breathing.

Methods: This is a prospective observational study of severe, critically ill adult COVID-19 patients admitted to the intensive care unit. All vital parameters were recorded in real time for all patients. Moreover, the results of chest computed tomography (CT), when available, were analyzed.

Results: PP was applied in 21 patients who were breathing spontaneously. The application of $\mathrm{PP}$ was associated with a significant increase in oxygen saturation measured by pulse oximetry $\left(\mathrm{SpO}_{2}\right)$ from $82 \% \pm 12 \%$ to $96 \% \pm 3 \%(\mathrm{P}<0.001) 1$ hour later. Moreover, $\mathrm{PP}$ was associated with a significant reduction in respiratory rate from $31 \pm 10$ to $21 \pm 4$ breaths/min $(P<0.001)$. Furthermore, the number of patients who exhibited signs of respiratory distress after PP was reduced from $10(47 \%)$ to $3(14 \%)(P=0.04)$. Early PP application also led to a clear improvement on $\mathrm{CT}$ imaging. It was not, however, associated with a reduction in mortality rate or in the use of invasive mechanical ventilation ( $\mathrm{P}>0.05$ for both).

Conclusions: Our study confirmed that the early application of PP can improve hypoxemia and tachypnea in COVID-19 patients with spontaneous breathing. Randomized controlled trials are needed to confirm the beneficial effects of PP in COVID-19 patients with spontaneous breathing.

Key Words: COVID-19; intensive care unit; patient outcomes; prone position; respiratory distress

\section{INTRODUCTION}

A newly identified coronavirus disease 2019 (COVID-19) caused by a severe acute respiratory syndrome coronavirus 2 (SARS-CoV-2) strain rapidly spread across China and other neighboring countries in early 2020. To date, the COVID-19 coronavirus has affected 219 countries, with more than 99 million infected patients leading to more than 3.7 million deaths [1]. Fever and cough are the most prevalent symptoms, while gastrointestinal symptoms are not as common [2]. However, severe COVID-19 infections are generally characterized by acute respiratory distress, and refractory hypoxemia is the most frequently reported cause of death $[2,3]$. Severe cases with acute respiratory distress requiring admission to an intensive care unit

\section{Original Article}

Received: April 22, 2021

Revised: June 8, 2021

Accepted: June 16, 2021

Corresponding author

Mabrouk Bahloul

Department of Intensive Care, Habib Bourguiba University Hospital, Sfax

3029, Tunisia

Tel: +216-98-69-8267

Fax: +216-74-24-3427

E-mail:bahloulmab@yahoo.fr

Copyright @ 2021 The Korean Society of Critical Care Medicine

This is an Open Access article distributed under the terms of Creative Attributions Non-Commercial License (https:// creativecommons.org/li-censes/by-nc/4.0/ which permits unrestricted noncommercial use, distribution, and reproduction in any medium, provided the original work is properly cited. 
(ICU) necessitate treatment via a high-flow nasal oxygen (HFNO) cannula or a facial mask.

On the other hand, non-invasive and/or invasive mechanical ventilation as supportive care is used for serious cases with severe hypoxemia. Recently, the prone position (PP) has also been used in the management of non-intubated COVID-19 patients with acute respiratory failure [4-6]. Despite the insufficiency of the data published about this subject [4-6], studies have suggested that early use of the PP in the management of acute respiratory failure due to COVID-19 infection can improve oxygenation and prevent the need for invasive mechanical ventilation. It can also help decrease the number of COVID-19 patients who ultimately die due to severe hypoxemia $[5,6]$. However, this result has not been confirmed by other studies $[4,7]$. In our ICU, the PP is usually used to treat non-intubated COVID-19 patients and hypoxemic acute respiratory failure. In this study, we explored whether early application of the PP (PP within 6 hours after ICU admission) can improve severe hypoxemia and respiratory failure in COVID-19 patients with spontaneous breathing.

\section{MATERIALS AND METHODS}

\section{Study Design and Setting}

This study was approved by the local Institutional Review Board of Habib Bourguiba University Hospital (IRB No. 310/2021) and the need for written informed consent was waived by the ethics committee. This a prospective observational study of severe, critically ill adult COVID-19 patients-all of whom had a confirmed SARS-CoV-2 infection-admitted into the ICU of a university hospital between September 1 and December 4, 2020 , for acute respiratory distress. The positive diagnosis of SARS-CoV-2 infection was confirmed by a reverse transcription polymerase chain reaction test in all cases. In this study, we included all patients with spontaneous breathing and whose hypoxemia (oxygen saturation measured by pulse oximetry $\left[\mathrm{SPO}_{2}\right]<92 \%$ ) did not resolve despite supplemental oxygen delivered via facial mask or HFNO cannula, and who accepted the PP. We excluded all patients who were admitted in cardiac arrest, those who required non-invasive and/or invasive mechanical ventilation on ICU admission, those with hemodynamic instability (shock), and those with neurological disorders (agitation and/or coma). However, patients who refused the PP were used as a control group.

Data collected for the included patients comprised epidemiological, clinical, laboratory, radiological, and treatment data on the day of admission and during ICU stay. In our ICU,

\section{KEY MESSAGES}

- Early application of prone position (PP) can improve severe hypoxemia and tachypnea in coronavirus disease 2019 (COVID-19) patients with spontaneous breathing.

- Early application of PP does not seem to be associated with a reduction in the use of invasive mechanical ventilation.

- Early application of PP does not seem to be associated with a reduction in mortality rate in COVID-19 patients with spontaneous breathing.

- Randomized controlled trials are needed to confirm the beneficial effects of PP in COVID-19 patients with spontaneous breathing.

corticosteroids (dexamethasone: 12-24 mg/day) were used to treat all SARS-CoV-2 infected patients. Moreover, zinc, vitamin $\mathrm{D}$ and vitamin $\mathrm{C}$, diuretics (in the absence of hypovolemia and/or shock), and enoxaparin were administered to all patients. In our ICU, illness severity was evaluated based on Simplified Acute Physiology Score (SAPS) II [8] and Sequential Organ Failure Assessment (SOFA) score [9]. All included patients were asked to take a PP for 2 to 4 hours (as tolerated by the patient) followed by 2 hours of supine positioning during the day, and to sleep in a PP at night, when possible.

Vital signs were monitored by a cardio-respiratory monitor and all vital parameters (heart rate, blood pressure, respiratory rate $[\mathrm{RR}]$, and $\mathrm{SpO}_{2}$ ) were recorded in real time for all patients. For all included patients, the last parameters were recorded 30 minutes before and 60 minutes after the application of the PP. Moreover, the results of chest computed tomography (CT), when performed, were analyzed. For rare patients, a PP chest CT was performed if possible (in our institution, CT is not always available). The primary outcome was the change in $\mathrm{SpO}_{2}, \mathrm{RR}$, and the evolution of signs of respiratory distress (such as retractions, accessory muscle use) prior to and 60 minutes after the application of the PP, without change in inspired oxygen concentration. The secondary outcome was the need for mechanical ventilation and the prognostic impact (i.e., wether the patient survived or died) of the PP.

\section{Statistical Modeling}

All statistical analyses were performed using IBM SPSS ver. 20.0 (IBM Corp., Armonk, NY, USA). Categorical data were expressed in proportion and subgroups, while continuous variables were expressed as mean ( \pm standard deviation). Comparisons between the PP group and the PP-free group (17 pa- 
tients who refused the PP) and of change in respiratory parameters $\left(\mathrm{SpO}_{2}, \mathrm{RR}\right.$, retractions) before and after $\mathrm{PP}$, were analyzed by the chi-square test (or Fisher's exact test), the twogroup t-test, or the Mann-Whitney U-test, as appropriate. The level of significance was set at $\mathrm{P}<0.05$.

\section{RESULTS}

From September 1, 2020, to December 4, 2020, a total of 96 patients were admitted to our ICU for respiratory distress due to a COVID-19 infection. Fifty-eight patients were excluded from this study because they had developed shock and/or required mechanical ventilation (invasive and/or noninvasive) on ICU admission. Moreover, in 17 cases, the PP was refused by patients. As a result, the PP was applied in only 21 patients with spontaneous breathing and they were all included. There were 16 male and 5 female patients. The mean age was $61.4 \pm 9.5$ with a median of 64 years. Body mass index (BMI) was more than $30 \mathrm{~kg} / \mathrm{m}^{2}$ in 10 cases (47.6\%). Mean SAPS II on ICU admission was $28.6 \pm 9.7$ and mean SOFA score was $4 \pm 1$.7. Moreover, 16 patients $(76 \%)$ had one or more pathological antecedents. The most common past medical conditions included arterial hypertension in 10 patients $(47.6 \%)$ and diabetes mellitus in 7 (33\%). A history of malignancy, structural lung diseases such as bronchiectasis, or immunocompromise such as human immunodeficiency virus (HIV) infection or autoimmune diseases were not present in any patient.

The most common symptoms on hospital admission were dyspnea, which was observed in 21 patients (100\%), cough in $15(71.4 \%)$, and fever $\left(\geq 38^{\circ} \mathrm{C}\right)$ in $13(62 \%)$. The mean time from

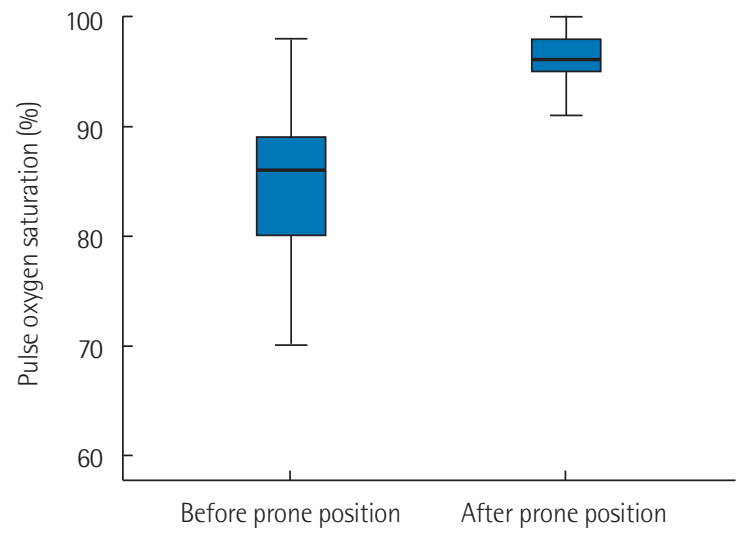

Figure 1. Evolution of oxygen saturation measured by pulse oximetry $\left(\mathrm{SpO}_{2}\right) 1$ hour after prone positioning. Early prone position improve significantly $\mathrm{SpO}_{2}$. Black line, median; box, 25\%-75\%; error bar, range. the onset of symptoms to ICU admission was $9.7 \pm 5.8$ days. The cause of ICU admission was acute respiratory failure in all cases. On ICU admission, all patients exhibited major signs of respiratory distress. The mean RR was at $31 \pm 10$ breaths $/ \mathrm{min}$, the mean $\mathrm{SpO}_{2}$ was at $82 \% \pm 11 \%$ (under oxygen support via a facial mask or HFNO cannula). The mean body temperature was $37.8^{\circ} \mathrm{C} \pm 1.0^{\circ} \mathrm{C}$. Moreover, 10 patients (47.6\%) exhibited signs of respiratory distress. Oxygen support was required for all patients (100\%): via facial mask for 9 (43\%), HFNO for 12 (57\%). In our ICU, corticosteroids (dexamethasone: 12-24 $\mathrm{mg} /$ day) were used to treat all SARS-CoV-2 infected patients. However, remdesivir and hydroxychloroquine were not used for any patient. During their ICU stay, six patients (28.5\%) developed a hospital-acquired infection (ventilator-associated pneumonia in five patients and urinary tract infection in one patient), one patient (4.5\%) experienced a thromboembolic event, and six patients (28.5\%) experienced a kidney injury.

The mean arterial oxygen tension $\left(\mathrm{PaO}_{2}\right)$ /fractional inspired oxygen $\left(\mathrm{FiO}_{2}\right)$ ratio was $88 \pm 37$ with a median at 80 . The $\mathrm{PaO}_{2} /$ $\mathrm{FiO}_{2}$ ratio was under 200 in all patients except one. In the current study, the application of the PP in our patients was associated with a significant increase in the $\mathrm{SpO}_{2}$ from $82 \% \pm 12 \%$ to $96 \% \pm 3 \%(\mathrm{P}<0.001) 1$ hour after $\mathrm{PP}$ application (Figure 1$)$. Moreover, PP application was associated with a significant reduction in the RR from $31 \pm 10$ to $21 \pm 4$ breaths $/ \min (\mathrm{P}<0.001)$ (Figure 2). Additionally, the number of PP patients who exhibited signs of respiratory distress was reduced from 10 (47\%) to 3 (14\%) $(\mathrm{P}=0.04)$. Furthermore, early PP application led to clear improvement on CT (Figure 3). Mean ICU stay was $9.5 \pm 6.6$ days with a median at 9.5 days. During the ICU stay, nine patients $(43 \%)$ required invasive mechanical ventilation and 7

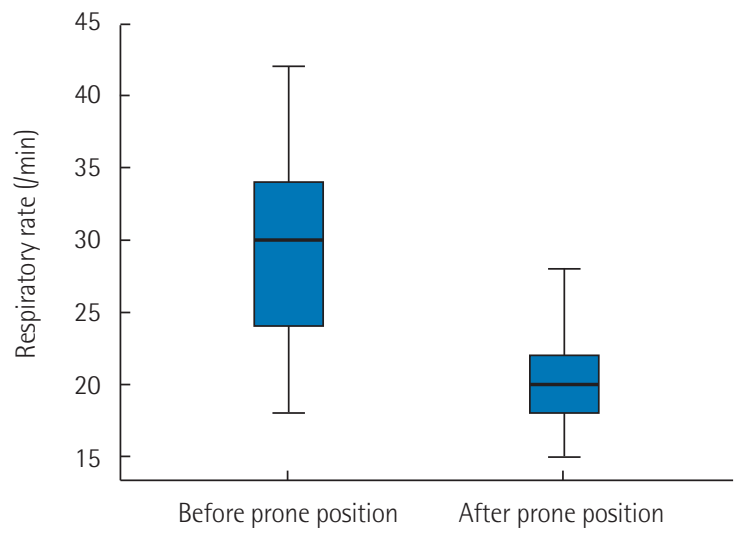

Figure 2. Evolution of respiratory rate 1 hour after prone positioning. Early prone position improve significantly respiratory rate. Black line, median; box, 25\%-75\%; error bar, range. 


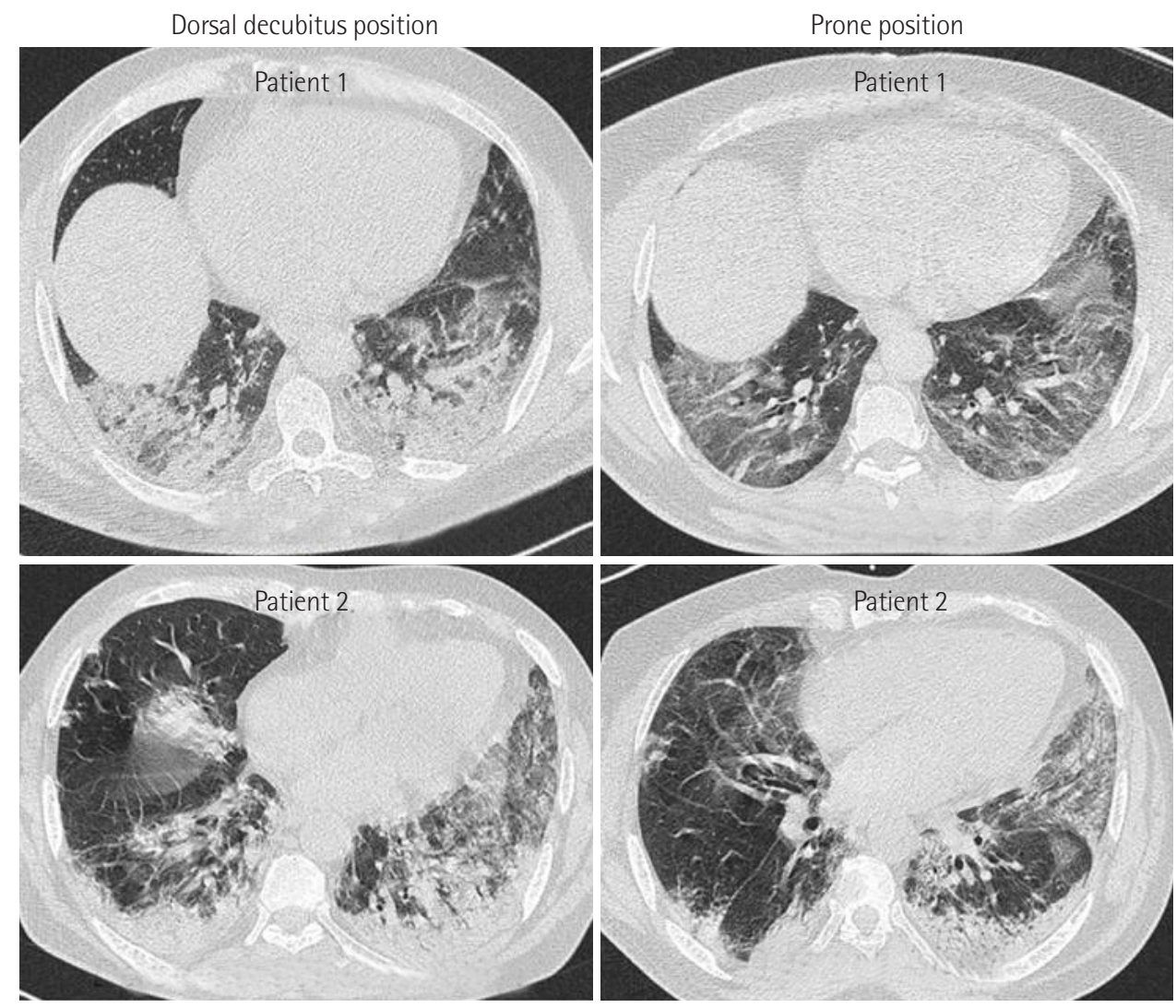

Figure 3. Computed tomography imaging showed that the density and scope of diffuse patch shadow and pulmonary condensation in both lungs were significantly improved after prone position.

\section{(33\%) died.}

Lastly, comparison between the PP-free group (17 patients who refused the PP) and the PP group showed that the two groups were comparable (Table 1). Chest CT was performed in 17 patients in the PP group and in eight patients in the control group with no difference in terms of severity between the two groups (Table 1). Moreover, early application of the PP was not associated with a reduction in mortality rate or in the use of invasive mechanical ventilation ( $\mathrm{P}>0.05$ for both) (Table 1). Finally, the time from ICU admission to the use of mechanical ventilation was longer in patients in the PP group in comparison with the control group ( $8.1 \pm 5.8$ vs. $6.0 \pm 3.2$ days, $\mathrm{P}=0.17)$.

\section{DISCUSSION}

Our study confirmed that early application of the PP can improve severe hypoxemia and respiratory failure in COVID-19 patients with spontaneous breathing. In fact, early application of the PP in these patients led to a significant increase in $\mathrm{SpO}_{2}$, a significant reduction in $\mathrm{RR}$, and a significant reduction in signs of respiratory distress. Nevertheless, we found that, in comparison with the control group (PP-free group), early application of PP was not associated with a reduction in mortality rate or in the use of invasive mechanical ventilation. However, we found that invasive mechanical ventilation application was delayed longer in patients in the PP group in comparison with the control group.

Although we were surprised by the absence of a prognostic impact (i.e., wether the patient survived or died) of early PP in COVID-19 patients, similar results have recently been published $[4,7]$. In fact, in a prospective, multicenter, observational cohort study in consecutive COVID-19 patients with acute respiratory failure requiring respiratory support with HFNO, Ferrando et al. [4] found that the use of awake-PP as adjunctive therapy to HFNO did not reduce the risk of intubation. Moreover, although the use of awake-PP with HFNO showed a trend toward delaying intubation compared to HFNO alone, early use of awake-PP did not affect 28-day mortality (RR, 1.04; 95\% confidence interval [CI], 0.40-2.72; $\mathrm{P}=0.92$ ). However, some other studies showed that the use of early PP to treat COVID-19 patients with severe hypoxia improves hypoxemia, 
Table 1. The clinical presentation of the study group on hospital admission and on the day of ICU admission

\begin{tabular}{|c|c|c|c|}
\hline Variable & PP group $(n=21)$ & PP-free group $(n=17)$ & P-value \\
\hline \multicolumn{4}{|l|}{ Demographic characteristics } \\
\hline Age (yr) & $61 \pm 10$ & $60 \pm 12$ & 0.68 \\
\hline Obesity (BMI > 30 kg/m²) & 10 & 4 & 0.18 \\
\hline Diabetes mellitus & 7 & 7 & 0.74 \\
\hline Arterial hypertension & 10 & 6 & 0.52 \\
\hline COPD & 2 & 0 & 0.49 \\
\hline \multicolumn{4}{|l|}{ Symptom on hospital admission } \\
\hline Dyspnea & 21 & 10 & 0.02 \\
\hline Cough & 15 & 6 & 0.48 \\
\hline Fever & 13 & 5 & 0.06 \\
\hline Headache & 5 & 1 & 0.19 \\
\hline Anosmia/ageusia & 3 & 2 & 0.60 \\
\hline Vomiting & 3 & 2 & 0.60 \\
\hline Abdominal pain & 5 & 0 & 0.05 \\
\hline Diarrhea & 4 & 0 & 0.11 \\
\hline \multicolumn{4}{|l|}{ Clinical findings on ICU admission } \\
\hline Respiratory rate/min & $31 \pm 10$ & $27 \pm 7$ & 0.16 \\
\hline $\mathrm{SpO}_{2}$ under oxygen & $83 \pm 11$ & $85 \pm 8$ & 0.41 \\
\hline Sign of respiratory fight & 10 & 5 & 0.32 \\
\hline SAPS II score & $28.6 \pm 9.7$ & $29.7 \pm 8.5$ & 0.73 \\
\hline SOFA score & $4.2 \pm 1.7$ & $4.4 \pm 1.7$ & 0.70 \\
\hline $\mathrm{pH}$ & $7.44 \pm 0.03$ & $7.43 \pm 0.03$ & 0.45 \\
\hline Blood bicarbonate (mmol/L) & $23.7 \pm 2.8$ & $24.3 \pm 4.9$ & 0.69 \\
\hline $\mathrm{PaO}_{2} / \mathrm{FiO}_{2}$ ratio & $88 \pm 37$ & $80 \pm 20$ & 0.45 \\
\hline GGOP $>50 \%$ on chest CT scan & $15 / 17^{\mathrm{a}}$ & $8 / 8^{a}$ & 0.45 \\
\hline \multicolumn{4}{|l|}{ Treatment and outcome } \\
\hline High-flow nasal oxygen & 12 & 4 & 0.05 \\
\hline Invasive mechanical ventilation & 9 & 4 & 0.30 \\
\hline Corticosteroid & 21 & 17 & 1 \\
\hline Diuretics & 19 & 10 & 0.05 \\
\hline ICU stay (day) & $9.5 \pm 6.6$ & $7.6 \pm 3.7$ & 0.13 \\
\hline Delay of invasive mechanical ventilation (day) & $8.1 \pm 5.8$ & $6.0 \pm 3.2$ & 0.17 \\
\hline Survivor & 7 & 5 & 0.79 \\
\hline
\end{tabular}

Values are presented as mean \pm standard deviation or number.

ICU: intensive care unit; PP: prone position; BMI: body mass index; COPD: chronic obstructive pulmonary disease; $\mathrm{SpO}_{2}$ : oxygen saturation measured by pulse oximetry: SAPS: Simplified Acute Physiology Score; SOFA: Sequential Organ Failure Assessment; $\mathrm{PaO}_{2}$ : arterial oxygen tension; FiO ${ }_{2}$ : fractional inspired oxygen; GGOP: ground glass opacity portion; CT: computed tomography.

${ }^{a}$ Total number of patients with chest CT scan.

prevents the need for invasive mechanical ventilation, and may be associated with a significant reduction in mortality rate $[5,6,10]$. In a single-center retrospective study including 48 patients treated for COVID-19 in the UK, Hallifax et al. [10] showed that PP of COVID-19 patients was associated with a significant reduction in mortality rate. Moreover, multivariate analysis showed that lack of awake-PP was an independent predictor of COVID-19 mortality (OR, 0.06; 95\% CI, 0.01-0.55). Thus, PP of COVID-19 patients appears to be associated with a significant improvement in hypoxemia. However, the prognos- 
tic impact (i.e., wether the patient survived or died) of this method on the mortality rate and on the need for invasive mechanical ventilation is not well established. In our study, early application of PP was also associated with a significant increase in $\mathrm{SpO}_{2}$, a significant reduction in the $\mathrm{RR}$, and a significant reduction in signs of respiratory distress. The mechanism of improvement of hypoxemia can be explained by the increase in lung recruitability and alveolar recruitment following PP, which causes ventilation-perfusion (V/Q) enhancement. Our results are in line with this hypothesis. CT imaging revealed that the density and scope of diffuse patch shadow and pulmonary condensation in both lungs were significantly improved by PP.

Oxygenation improvement, however, did not persist after return to the dorsal position; instead, it led to a new decrement and alveolar collapse. However, oxygenation improvement did not persist after returning to the dorsal position; instead, dorsal position led to a new decrement and alveolar collapse. This occurrence (i.e., decrement and alveolar collapse after return to the dorsal position) can explain why early application of PP was not associated with a reduction in the mortality rate or a decrease in the use of invasive mechanical ventilation. Moreover, the temporary improvement of oxygenation under PP, can explain the long time needed between ICU admission and the use of invasive mechanical ventilation in patients in the PP group in comparison with the control group.

Finally, our study suffers from some limitations. First, the number of included patients is small. Second, in our study $\mathrm{SpO}_{2}$ was analyzed instead of arterial blood gas analysis (ABGA) $\mathrm{PaO}_{2}$ in the primary outcomes and blood gases were not studied before and after the PP. We excluded all patients with hemodynamic instability (shock), which represents the most frequent source of error in pulse oximetry in this specific condition [11]. Moreover, no patients had dark skin pigmentation and there was no use of fingernail polish by the included female patients. Pulse oximetry has many advantages compared to $A B G$ monitoring. For instance, $A B G$ is an invasive, painful, and expensive procedure; ABGs seldom reflect venous samples; and measuring an ABG may delay interventions, such as invasive mechanical ventilation. For these reasons, $\mathrm{SpO}_{2}$ was used as a monitoring parameter in addition to other clinical parameters (RR, and the evolution of signs of respiratory distress) in our study. This type of methodology was previously used in a number of published studies [6,12-14]. Lastly, all included patients were not managed by the same team of physicians and did not receive the same therapies before ICU admission.
Our study confirms that early application of the PP can improve severe hypoxemia and respiratory failure in COVID-19 patients with spontaneous breathing. However, early application of PP was not associated with a reduction in mortality rate or in the use of invasive mechanical ventilation. Randomized controlled trials are needed to confirm the beneficial effects of PP in COVID-19 patients with spontaneous breathing.

\section{CONFLICT OF INTEREST}

No potential conflict of interest relevant to this article was reported.

\section{ORCID}

Mabrouk Bahloul https://orcid.org/0000-0002-6488-8294

\section{AUTHOR CONTRIBUTIONS}

Conceptualization: MB, SK, KBM, MB. Data curation: MB, SK, $\mathrm{MH}, \mathrm{OT}$, RA, BS, CBH, HC, KBM. Formal analysis: MB, SK, MH, AM, OT, KC, RA, BS, CBH. Methodology: MB, SK, AM, OT, KC, BS, CBH, HC, MB. Project administration: MB, BS, CBH. Visualization: $\mathrm{MB}, \mathrm{AM}, \mathrm{HC}$, KBM. Writing-original draft: $\mathrm{MB}, \mathrm{MH}$ $\mathrm{KC}, \mathrm{CBH}, \mathrm{HC}, \mathrm{MB}$. Writing-review \& editing: $\mathrm{MB}, \mathrm{SK}, \mathrm{CBH}$, $\mathrm{HC}$.

\section{REFERENCES}

1. Worldometer. Coronavirus update [Internet]. Worldometer; 2020 [cited 2020 Jul 24]. Available from: https://www.worldometers.info/coronavirus/.

2. Vetter P, Vu DL, L'Huillier AG, Schibler M, Kaiser L, Jacquerioz F. Clinical features of covid-19. BMJ 2020;369:m1470.

3. Bahloul M, Ketata W, Lahyeni D, Mayoufi H, Kotti A, Smaoui F, et al. Pulmonary capillary leak syndrome following COVID-19 virus infection. J Med Virol 2021;93:94-6.

4. Ferrando C, Mellado-Artigas R, Gea A, Arruti E, Aldecoa C, Adalia $\mathrm{R}$, et al. Awake prone positioning does not reduce the risk of intubation in COVID-19 treated with high-flow nasal oxygen therapy: a multicenter, adjusted cohort study. Crit Care 2020;24:597.

5. Coppo A, Bellani G, Winterton D, Di Pierro M, Soria A, Faverio $\mathrm{P}$, et al. Feasibility and physiological effects of prone positioning in non-intubated patients with acute respiratory failure due to COVID-19 (PRON-COVID): a prospective cohort study. Lancet Respir Med 2020;8:765-74. 
6. Caputo ND, Strayer RJ, Levitan R. Early self-proning in awake, non-intubated patients in the emergency department: a single ED's experience during the COVID-19 pandemic. Acad Emerg Med 2020;27:375-8.

7. Telias I, Katira BH, Brochard L. Is the prone position helpful during pontaneous breathing in patients with COVID-19? JAMA 2020;323:2265-7.

8. Le Gall JR, Lemeshow S, Saulnier F. A new Simplified Acute Physiology Score (SAPS II) based on a European/North American multicenter study. JAMA 1993;270:2957-63.

9. Ferreira FL, Bota DP, Bross A, Mélot C, Vincent JL. Serial evaluation of the SOFA score to predict outcome in critically ill patients. JAMA 2001;286:1754-8.

10. Hallifax RJ, Porter BM, Elder PJ, Evans SB, Turnbull CD, Hynes $\mathrm{G}$, et al. Successful awake proning is associated with improved clinical outcomes in patients with COVID-19: single-centre high-dependency unit experience. BMJ Open Respir Res 2020; 7:e000678.

11. Luks AM, Swenson ER. Pulse oximetry for monitoring patients with COVID-19 at home: potential pitfalls and practical guidance. Ann Am Thorac Soc 2020;17:1040-6.

12. Venus K, Munshi L, Fralick M. Prone positioning for patients with hypoxic respiratory failure related to COVID-19. CMAJ 2020;192:E1532-7.

13. Damarla M, Zaeh S, Niedermeyer S, Merck S, Niranjan-Azadi A, Broderick B, et al. Prone positioning of nonintubated patients with COVID-19. Am J Respir Crit Care Med 2020;202: 604-6.

14. Ponnapa Reddy M, Subramaniam A, Afroz A, Billah B, Lim ZJ, Zubarev A, et al. Prone positioning of nonintubated patients with coronavirus disease 2019: a systematic review and meta-analysis. Crit Care Med 2021 Apr 30 [Epub]. https://doi. org/10.1097/CCM.0000000000005086. 